\title{
hSav1 interacts with HAX1 and attenuates its anti-apoptotic effects in MCF-7 breast cancer cells
}

\author{
XUELAI LUO ${ }^{1 *}$, ZHAOMING LI ${ }^{1 *}$, XIAOLAN LI ${ }^{2}$, GUIHUA WANG ${ }^{1}$, WEICHENG LIU ${ }^{1}$, SUO DONG ${ }^{1}$,

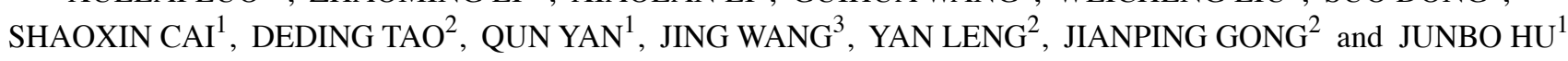 \\ Departments of ${ }^{1}$ Surgery and ${ }^{2}$ Molecular Medicine Center, Tongji Hospital, ${ }^{3}$ Department of Immunology, \\ Tongji Medical College, Huazhong University of Science and Technology, Wuhan 430030, P.R. China
}

Received February 1, 2011; Accepted March 4, 2011

DOI: $10.3892 /$ ijmm.2011.692

\begin{abstract}
It has been reported that Salvador (SAV) is a core component of the Salvador-Warts-Hippo (SWH) pathway that restricts cell number, by functioning as a dual regulator of cell proliferation and apoptosis in Drosophila. However, the function of its human ortholog hSav1 (also called hWW45) in mammalian cells is poorly understood. In this study, we identified hematopoietic cell-specific protein 1 (HS1)-associated protein X-1 (HAX1), a 35-kDa protein localized to cell mitochondria, as a novel binding partner of hSav1 using a yeast two-hybrid screening technique. Our finding was confirmed by immunoprecipitation and glutathione-S-transferase (GST) pull-down assays of both proteins. Using immunofluorescence staining, we showed that HAX1 and hSav1 interact with each other. Analysis of the anti-apoptotic function of HAX1 revealed that the presence of hSav1 attenuated the HAX1 protective effects from hydrogen peroxide $\left(\mathrm{H}_{2} \mathrm{O}_{2}\right)$-induced cell death in MCF-7 cells, while knockdown of hSav1 by small interfering RNAs (siRNAs) significantly enhanced the anti-apoptotic function of HAX1. Also, using the Oncomine database, we found several studies in which HAX1 levels were significantly up-regulated and hSav1 expression was down-regulated in breast cancer samples compared to normal breast tissue. In summary, we conclude that hSav1 interacts with HAX1 and attenuates its protective role against apoptosis in MCF-7 breast cancer cells.
\end{abstract}

Correspondence to: Dr Junbo Hu, Department of Surgery, Tongji Hospital, Tongji Medical College, Huazhong University of Science and Technology, Wuhan 430030, P.R. China

E-mail: jbhu@tjh.tjmu.edu.cn

Dr Jianping Gong, Molecular Medicine Center, Tongji Hospital, Tongji Medical College, Huazhong University of Science and Technology, Wuhan 430030, P.R. China

E-mail:.jpgong@tjh.tjmu.edu.cn

*Contributed equally

Key words: hematopoietic cell-specific protein 1-associated protein $\mathrm{X}-1, \mathrm{hSav1}$, apoptosis

\section{Introduction}

Apoptosis is a form of cell death with critical functions both in the development of organisms and in maintaining cellular homeostasis throughout life. Many signaling pathways control these important cellular processes, including the recently described Salvador-Warts-Hippo (SWH) pathway, which includes several tumor suppressor genes, such as: fat $(1,2)$ merlin (3), expanded, hippo (4), RASSF (5), Lats/Warts (6), Salvador (7) and an oncogene called yorkie (8-10) and controls cell growth, proliferation, and apoptosis (10). The ortholog of hSav1 in Drosophila, Salvador (SAV), has been reported as a core component of the SWH pathway.

SAV is a scaffold protein containing a WW domain that interacts with Warts (WTS) and a C-terminal SAV-RASSF-HPO (SARAH) domain that mediates binding to Hippo (HPO) (11). The primary function of SAV is likely to facilitate the close association of WTS and HPO kinases, and/or to recruit HPO to its site of activation (12-14). SAV promotes both cell cycle exit and apoptosis through the modulation of both cyclin $\mathrm{E}$ and DIAP (Drosophila inhibitor of apoptosis protein) in Drosophila. However, the cellular function of hSavl in mammalian cells is poorly understood. To better understand the mechanistic function of hSav1, it is necessary to identify new substrates for this protein. In the present study, we investigated the interaction between the hematopoietic cell-specific protein 1 (HS1)-associated protein X-1 (HAX1) and hSav1, which has been examined in previous studies (15).

HAX1 is a $35-\mathrm{kDa}$ anti-apoptotic protein that has sequence similarity to the Nip3 protein and shares homology with the $\mathrm{BH} 1$ and $\mathrm{BH} 2$ domains from the Bcl-2 family of proteins (15). HAX1 was originally identified in a yeast two-hybrid assay on the basis of its binding to HS1. The HAX1 protein appears to be expressed ubiquitously in various tissues and is localized mainly in mitochondria, but is also found in the endoplasmic reticula and nuclear envelopes of cells. HAX1 is involved in both death receptor and mitochondria-mediated apoptosis pathways. Previous studies showed that HAX1 is a potent inhibitor of Bcl-2-associated X protein (Bax)-induced apoptosis (16). Furthermore, it has been demonstrated that HAX1 is degraded in the mitochondria by the high temperature requirement protein A2 (Omi/HtrA2) after induction of apoptosis, which contributes to caspase-independent induction of apoptosis by 
Omi/HtrA2 (17). An additional study showed both that HAX1 is overexpressed in psoriatic skin using in situ hybridization and that keratinocytes isolated from psoriatic plaques, which are characterized by hyperproliferation and resistance to apoptosis, also overexpressed HAX1 (18). Recently, it was reported that HAX1 is an anti-apoptotic molecule that protects cardiac myocytes from hypoxia/reoxygenation-induced apoptosis by inhibiting caspase-9 $(19,20)$. Furthermore, the authors showed that HAX1 deficiency causes Kostmann disease, a severe, autosomal, recessive type of congenital neutropenia. Although many studies have touched upon the various roles of HAX1, certain questions remain unanswered. In this study, we aimed to demonstrate that HAX1 is a novel binding partner of hSav1.

\section{Materials and methods}

Reagents. The reagents we used in this study are as follows: Dulbecco's modified Eagle's medium (DMEM) and fetal bovine serum (FBS) were purchased from Gibco (Carlsbad, CA, USA). Monoclonal antibodies specific for hemagglutinin (HA) were purchased from Sigma-Aldrich (St. Louis, MO, USA). Anti-hSavl monoclonal antibody was purchased from Abnova (Taipei City, Taiwan, China). Cleaved poly-(ADPribose) (PARP) and cleaved caspase-9 were purchased from Cell Signaling Technology (Danvers, MA, USA). Horseradish peroxidase-conjugated secondary antibodies (anti-rabbit or anti-mouse IgG) and FITC- and TRITC-conjugated goat antibodies to mouse or rabbit IgG were purchased from Pierce (Rockford, IL, USA). Anti-HAX1 monoclonal antibody and an Annexin $\mathrm{V}$ and propidium iodide (Annexin V/PI) kit was purchased from BD Biosciences (San Diego, CA, USA). Protein A Sepharose ${ }^{\mathrm{TM}} \mathrm{CL}-4 \mathrm{~B}$ was purchased from Amersham Biosciences AB (Uppsala, Sweden). Other chemical reagents were purchased from Generay Biotech (Wuhan, China) or Tiangen Co. (Wuhan, China), unless otherwise noted.

Plasmid construction. Full-length HAX1 was isolated from a human, fetal liver, cDNA library and cDNA was subcloned into pCMV-FLAG4, pEGFP-N1 and pGEX-4T1 plasmids. HAX1 mutants were also generated by polymerase chain reaction (PCR) and cloned into the pGEX-4T1 plasmid. Similarly, cDNA for hSavl was obtained from a human, fetal liver, cDNA library, but was subcloned into pCMV-HA, pEGFP-N1 and pGEX-4T1 plasmids. Sequence verified constructs were used in all experiments.

Cell culture and transfection. HEK293 and MCF-7 cells were cultured in DMEM supplemented with $10 \%$ FBS, $100 \mathrm{U} / \mathrm{ml}$ penicillin and $0.1 \mathrm{mg} / \mathrm{ml}$ streptomycin in a $5 \% \mathrm{CO}_{2}$ atmosphere at $37^{\circ} \mathrm{C}$. Transfections were performed using the Lipofectamine 2000 reagent, according to the manufacturer's instructions (Invitrogen, Carlsbad, CA, USA).

Small interfering RNA (siRNA) experiments. In order to down-regulate hSav1 using siRNAs, we purchased the following sequences from RiboBio Co. (Guangzhou, China): si-hSavl-sense, (5'-3')-CCAUGAUCUCUUCCAAAGAdTdT; antisense, (3'-5')-dTdTGGUACUAGAGAAGGUUUCU; scrambled siRNA was used as a control. Cells were seeded in 6-well plates and transfected with siRNA duplexes using
Lipofectamine 2000 (Invitrogen), according to the manufacturer's recommendations.

Yeast two-hybrid screen. Yeast two-hybrid screening was performed using the Matchmaker Two-Hybrid System 3, according to the manufacturer's protocol (Clontech, Palo Alto, CA, USA). Briefly, the open reading frame of wild-type human hSavl was fused to the DNA-binding domain of the yeast transcription factor GAL4 and used as bait after transformation into the Saccharomyces cerevisiae strain AH109. To screen for proteins that interact with hSav1, the human fetal liver library was fused to the transcription-activating domain of GAL4 (Clontech). Positive clones were amplified and plasmids were rescued and sequenced to identify the interacting partner.

Western blot analysis and immunoprecipitation. Cell lysates were extracted into a cold lysis buffer [Tris- $\mathrm{HCl} \mathrm{pH} 7.5$, $150 \mathrm{mM} \mathrm{NaCl}, 1 \mathrm{mM}$ EDTA, $1 \mathrm{mM} \mathrm{MgCl} 2,0.5 \%$ Triton X-100, phosphatase inhibitor mixture $\left(1 \mathrm{mM} \mathrm{NaF}, 1 \mathrm{mM} \mathrm{Na}_{3} \mathrm{VO}_{4}\right.$ and $1 \mathrm{mM} \beta$-glycerophosphate), and a protease inhibitor mixture $(1 \mathrm{mmol} / \mathrm{l}$ PMSF, $2 \mu \mathrm{g} / \mathrm{ml}$ aprotinin, $1 \mu \mathrm{g} / \mathrm{ml}$ leupeptin and $1 \mu \mathrm{g} / \mathrm{ml}$ pepstatin A)]. Lysates were clarified by centrifugation at $10,000 \mathrm{x} \mathrm{g}$ for $20 \mathrm{~min}$. For immunoprecipitation, the lysates were incubated with $1 \mu \mathrm{g}$ of primary antibodies for $2 \mathrm{~h}$ at $4^{\circ} \mathrm{C}$ with rocking, followed by protein A sepharose beads for $4 \mathrm{~h}$ at $4^{\circ} \mathrm{C}$. The bound immunocomplexes were washed six times with cold lysis buffer, and eluted into $60 \mu 1$ 1X SDS, by boiling the samples for $5 \mathrm{~min}$. The supernatant was collected after centrifugation at $4000 \times \mathrm{g}$ for $5 \mathrm{sec}$. The samples were resolved on an SDS-PAGE gel and transferred to a PVDF membrane (Amersham Biosciences). The membrane was blocked in TBS-T buffer (20 mM Tris- $\mathrm{HCl}, \mathrm{pH} 7.5,150 \mathrm{mM}$ $\mathrm{NaCl}$ and $0.05 \%$ Tween-20) containing 5\% (w/v) non-fat milk at room temperature for $1 \mathrm{~h}$ and then incubated with the primary antibody overnight at $4{ }^{\circ} \mathrm{C}$, followed by incubation with horseradish peroxidase-conjugated anti-IgG for $1 \mathrm{~h}$ at room temperature. Bands were visualized using a SuperSignal West Femto Maximum Sensitivity Substrate Trial kit (Pierce).

Generation and purification of glutathione-S-transferase (GST) proteins, GST pull-down assay. GST proteins were induced with $0.1 \mathrm{mM}$ isopropyl- $\beta$-d-thiogalactopyranoside (IPTG) for $3 \mathrm{~h}$ after transforming the plasmids into Escherichia coli DH10B (Invitrogen). Proteins were purified after affinity chromatography on glutathione Sepharose ${ }^{\mathrm{TM}}$ 4B (Amersham Biosciences). HEK293 cells were transiently transfected with the indicated expression plasmids and harvested $36 \mathrm{~h}$ after transfection. GST pull-down was performed by incubating $1 \mathrm{mg}$ of whole-cell extracts with equal amounts of recombinant GST and GST-HAX1 or HAX1 mutants at $4^{\circ} \mathrm{C}$ overnight. The beads were washed three times with $10 \mathrm{mM} \mathrm{NaPO}_{4}(\mathrm{pH} 7.2)$, $10 \mathrm{mM} \mathrm{NaN}_{3}, 120 \mathrm{mM} \mathrm{NaCl}, 0.1 \%$ (v/v) Tween-20 mixture at $4^{\circ} \mathrm{C}$ and were suspended in $2 \mathrm{X}$ SDS sample buffer. Samples were analyzed by Western blot analysis.

Immunofluorescence. Cells grown on chamber slides were transfected with indicated plasmids and fixed with $4 \%$ formaldehyde in phosphate-buffered saline (PBS) for $10 \mathrm{~min}$. When MitoTracker was used, it was added to the cell culture for $30 \mathrm{~min}$ before fixation. Cells were permeabilized in PBS 
A

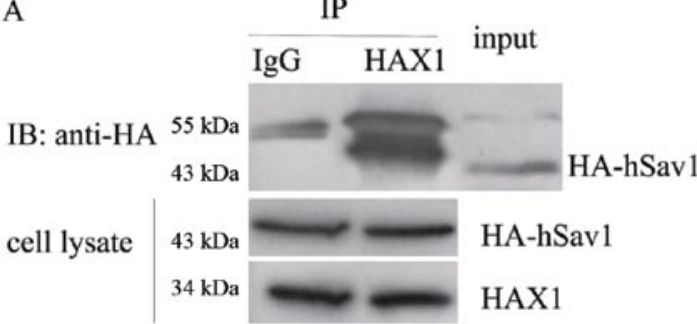

D
C
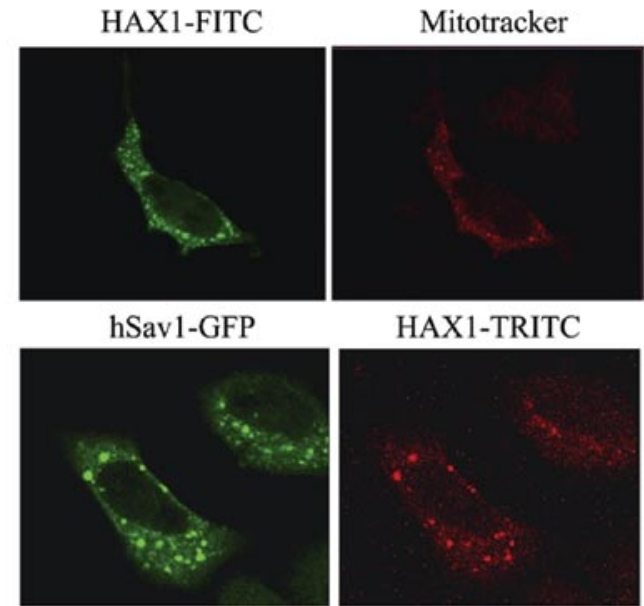

HAX1-TRITC

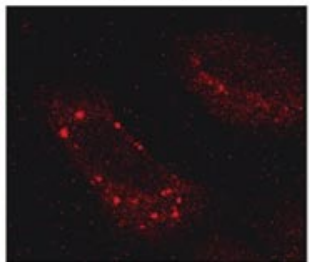

B

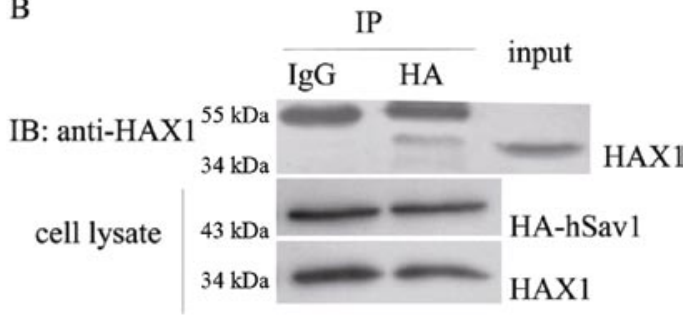

Merge

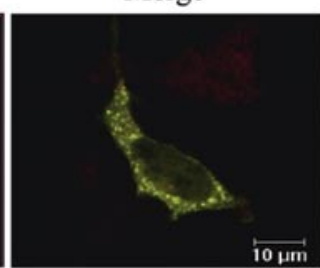

Merge

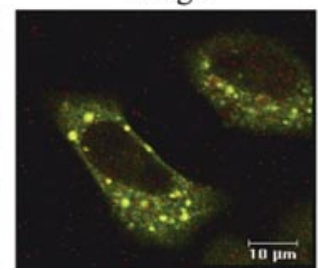

Figure 1. Interaction between hSav1 and HAX1 in vivo. (A and B) HEK293 cells were transiently transfected with expression plasmids encoding hemagglutinintagged hSav1. Thirty-six hours after transfection, whole-cell lysates were immunoprecipitated (IP) with (A) anti-hemagglutinin tag or (B) anti-HAX1 and anti-IgG antibodies. The immunoprecipitates were analyzed by immunoblotting (IB) with (A) anti-HAX1 and (B) anti-hemagglutinin tag antibodies. (C) HAX1 is mainly localized in the mitochondria. MCF-7 cells were stained with MitoTracker Red to visualize mitochondria (red) and anti-HAX1 monoclonal antibody followed by FITC-conjugated anti-mouse IgG to visualize HAX1 (green). (D) hSav1 co-localizes with HAX1. MCF-7 cells were transfected with hSav1-GFP, as previously described. After $36 \mathrm{~h}$, cells were fixed and immunostained with anti-HAX1 antibody followed by TRITC-conjugated anti-mouse IgG (red). Cells were visualized using confocal laser scanning microscopy. Co-localization of hSav1 and HAX1 yields a yellow color after merging the images.

containing $0.1 \%$ Triton $\mathrm{X}-100$ for $10 \mathrm{~min}$, and blocked with $2 \%$ bovine serum albumin prepared in PBS for $10 \mathrm{~min}$. Cells were then incubated with anti-hSav1 or anti-HAX1 monoclonal antibodies $(1 \mu \mathrm{g} / \mathrm{ml})$ for $1 \mathrm{~h}$. FITC- or TRITC-conjugated goat antibodies to mouse $\operatorname{IgG}$ were used at a dilution of 1:100 for 45 min. Cells were washed with PBS and nuclei were stained with PI $(10 \mu \mathrm{g} / \mathrm{ml})$ for $15 \mathrm{~min}$. Images were captured using a Leica confocal laser scanning microscope (Leica Laser Technik $\mathrm{GmbH}$, Heidelberg, Germany).

Assessment of cell death. After transfecting cells with the indicated plasmids (24-48 h), cells were mixed with the pro-apoptotic agent $\mathrm{H}_{2} \mathrm{O}_{2}(2 \mathrm{mM})$ for $15 \mathrm{~h}$. Apoptotic cells were quantified using Sub-G1- and Annexin V/PI-based flow cytometry, as described previously $(21,22)$.

Databases. We used the Oncomine cancer microarray database (23) (http://www.oncomine.org) to study the gene expression of HAX1 and hSav1 in human breast tumor types and their normal tissue counterparts (24-29). In order to compare the gene expression in a tumor type to its normal counterpart, gene expression data from a same study, performed with the same methodology, were used. The gene expression data were log transformed, median centered per array, and the standard deviation was normalized to one per array. A gene was considered as overexpressed when its mean value in tumor samples was significantly higher to its mean value in the normal tissue counterpart using a Student's t-test $(\mathrm{P}<0.05)$.
Statistical analysis. All data were expressed as the mean \pm SEM (standard error of the mean). Between-group and amonggroup comparisons were conducted using Student's t-tests and ANOVA, respectively. Differences were considered significant when $\mathrm{P}<0.05$.

\section{Results}

Interaction between hSavl and HAXl in vivo. To identify proteins that interact with hSavl, we used a yeast two-hybrid system. Of a total of $1.23 \times 10^{6}$ clones screened under highstringency conditions, 30 positive clones were identified. Three of these clones contained the full-length or a part of the cDNA sequence of HAX1. To confirm this interaction, co-immunoprecipitation experiments were carried out in HEK 293 cells. HEK 293 cells were transfected with a construct encoding the full-length hSav1 fused to a hemagglutinin (HA) tag. Anti-HA tag antibody was used to precipitate HA-tagged hSav1. Western blot analysis with HAX1 antibodies was used to monitor the presence of HAX1 protein in the precipitated complex. HA-tagged hSav1 co-precipitated with HAX1 from lysates of transiently transfected HEK293 cells, while it failed to co-precipitate from the control plasmid. Similarly, HAX1 endogenous to HEK293 cells co-precipitated with recombinant HA-tagged hSav1, but not with the control plasmid (Fig. 1A and B).

Further evidence that HAX1 and hSav1 interact in vivo was provided by immunofluorescence studies. HAX1, originally 
A

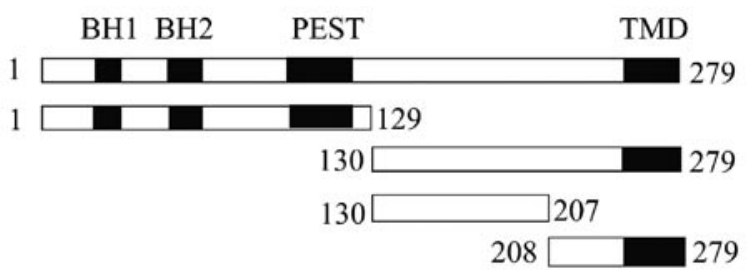

B

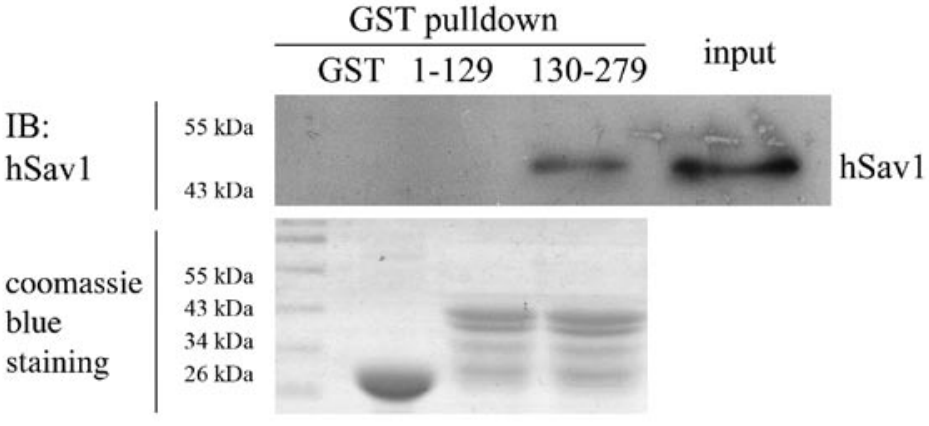

$\mathrm{C}$

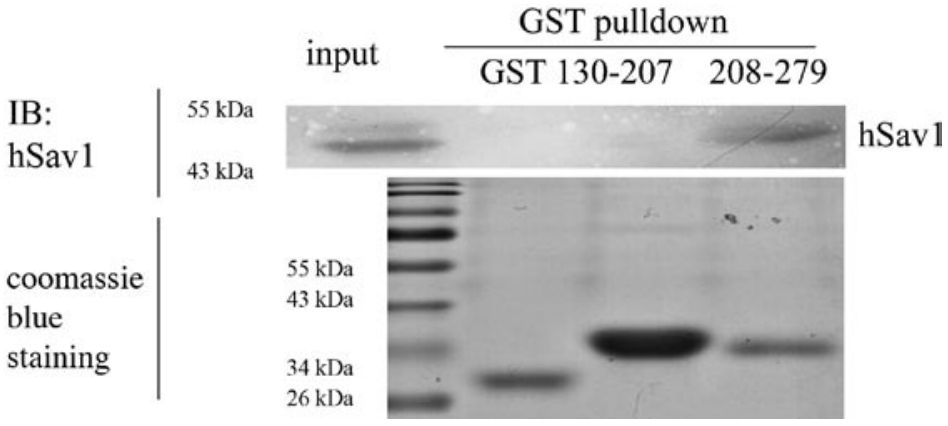

Figure 2. Association of hSav1 and HAX1 in vitro. (A) Schematic diagram of the functional domains of HAX1. (B and C) GST pull-down assays of hemagglutinin-tagged hSav1 with recombinant GST alone and GST fused with fragments of HAX1 (1-129, 130-279, 130-207 or 208-279). HEK293 cells were transiently transfected with hemagglutinin-tagged hSavl and lysates were mixed with the indicated GST fusions or GST alone. Complexes were captured with glutathione-sepharose and the bound protein was detected by hSavl immunoblot. IB, immunoblot.

isolated as an HS1 binding protein, is known to localize mainly in cellular mitochondria. In this study, we confirmed that HAX1 is mainly localized in the mitochondria of cells (Fig. 1C). We also examined whether HAX1 co-localizes with hSav1 in mammalian cells. Our results show that, in microscopy-derived images of cells, hSav1 (green) and HAX1 (red) co-localized, yielding a yellow color (Fig. 1D). Thus, we conclude that a substantial portion of HAX1 co-localizes with hSav1 in MCF-7 cells.

Association of hSavl and HAX1 in vitro. To verify if hSav1 and HAX1 directly interact with each other in vitro, we carried out GST pull-down assays. It is established that HAX1 contains BH1 (37-56), BH2 (74-89), PEST (104-117) and transmembrane domains (261-273). To investigate the importance of various related HAX1 domains in binding to hSav1, we generated deletion mutants lacking the various domains (Fig. 2A). The domains were expressed as GST-fusion proteins in E. coli and purified using glutathione sepharose. Equivalent amounts of either GST-HAX1 deletion mutants or control GST proteins bound to glutathione matrices were incubated with lysates of HEK293 cells, which were transiently transfected with a vector encoding a HA-tagged version of hSavl. The bound proteins were eluted and subjected to Western blot analysis (Fig. 2B and C). Results from these experiments showed that the HAX1 derived peptide corresponding to amino acids 208-279 of the fusion protein was sufficient for binding to hSavl. The GST protein alone did not bind to hSavl.

hSavl attenuates the anti-apoptotic function of HAX1. Previous reports have demonstrated an anti-apoptotic function for HAX1. In order to determine whether the presence of hSav1 may influence its anti-apoptotic role, we performed transient transfections of Flag-HAX1, HA-hSav1 or co-transfections of both constructs in MCF-7 cells. Cell death was induced by exposure to $2 \mathrm{mM} \mathrm{H}_{2} \mathrm{O}_{2}$ for $15 \mathrm{~h}$. At the end of the incubation period, cell death was determined by the Sub-G1 and Annexin V/PI flow cytometry assays. Cells co-transfected with the empty vectors were used as controls. Treatment with $\mathrm{H}_{2} \mathrm{O}_{2}$ induced $30 \%$ cell death in the control cells, whereas the HAX1 transfected cells displayed decreased cell death $(\sim 15 \%)$, compared to the control cells (Fig. 3A). However, the anti-apoptotic effect of HAX1 was attenuated when it was co-transfected with hSav1 $(\sim 30 \%$ cell death) $(\mathrm{n}=3$, $\mathrm{P}<0.05)$. Also, a same pattern was observed when we used the Annexin V/PI assay (Fig. 3B). HAX1 transfected cells showed a decreased cell death compared to control cells $(\sim 32$ vs. $\sim 57 \% ; n=3, P<0.05)$ and the anti-apoptotic effect of HAX1 was attenuated when it was co-transfected with hSavl $(\sim 32$ vs. $\sim 53 \% ; \mathrm{n}=3, \mathrm{P}<0.05)$. Next, we evaluated the anti-apoptotic 

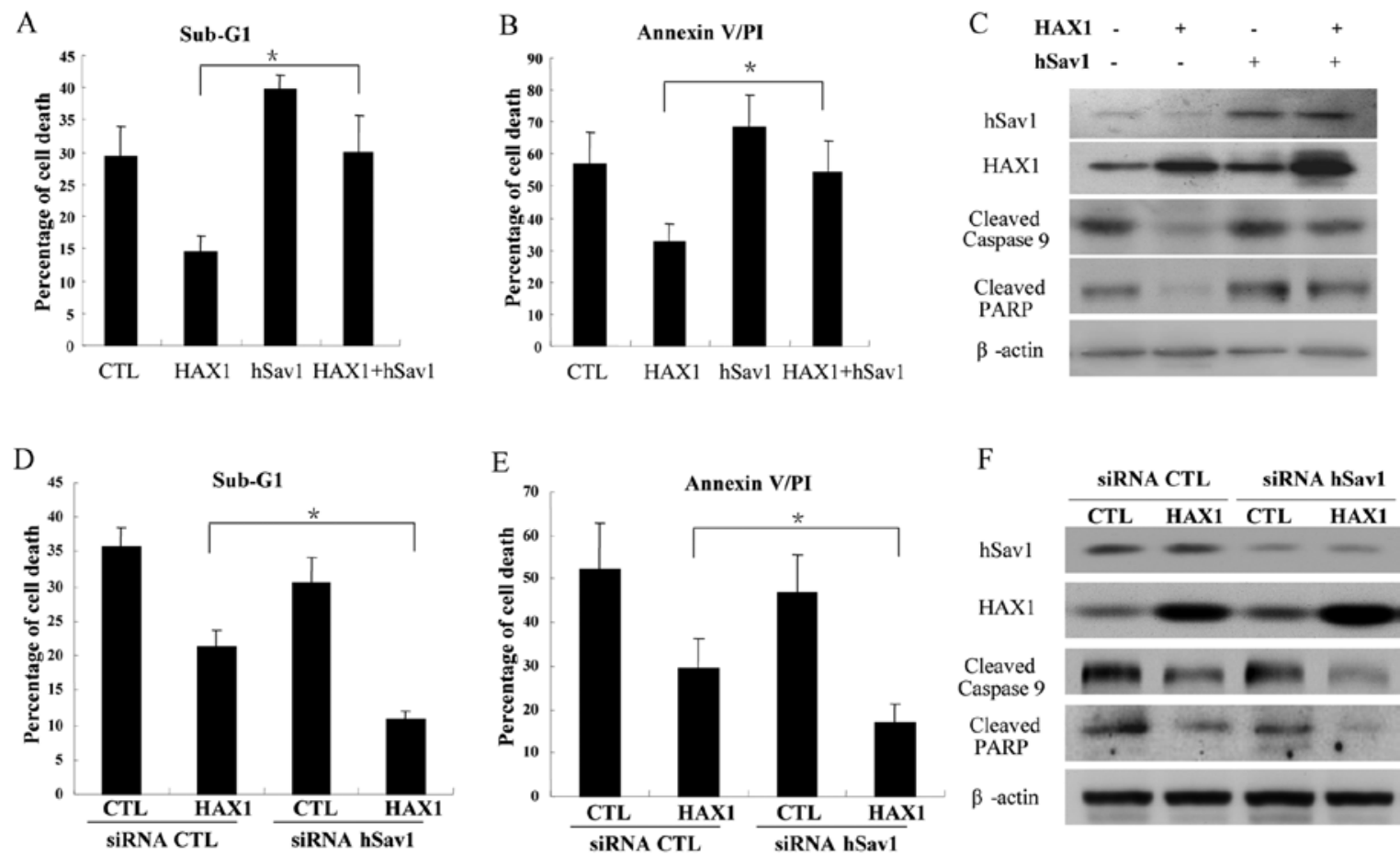

Figure 3. hSav1 attenuates the protective role of HAX1 in against apoptosis. (A-C) MCF-7 cells were transiently transfected with HA-hSav1 and/or Flag-HAX1. After $24 \mathrm{~h}$, the cells were treated with $\mathrm{H}_{2} \mathrm{O}_{2}(2 \mathrm{mM})$ for $15 \mathrm{~h}$. Apoptotic cells were quantified by (A) Sub-G1- and (B) Annexin V/PI-based flow cytometry and (C) Western blot analysis shows the protein changes. (D-F) MCF-7 cells were transiently transfected with Flag-HAX1 and/or hSav1 siRNA. After 60 h, cells were treated with $\mathrm{H}_{2} \mathrm{O}_{2}(2 \mathrm{mM})$ for $15 \mathrm{~h}$. After treatment, cell death was analyzed by (D) Sub-G1- and (E) Annexin V/PI-based flow cytometry and (F) Western blot analysis shows the subsequent protein changes. All experiments were performed in triplicate. ${ }^{*} \mathrm{P}<0.05$. CTL, control.

A
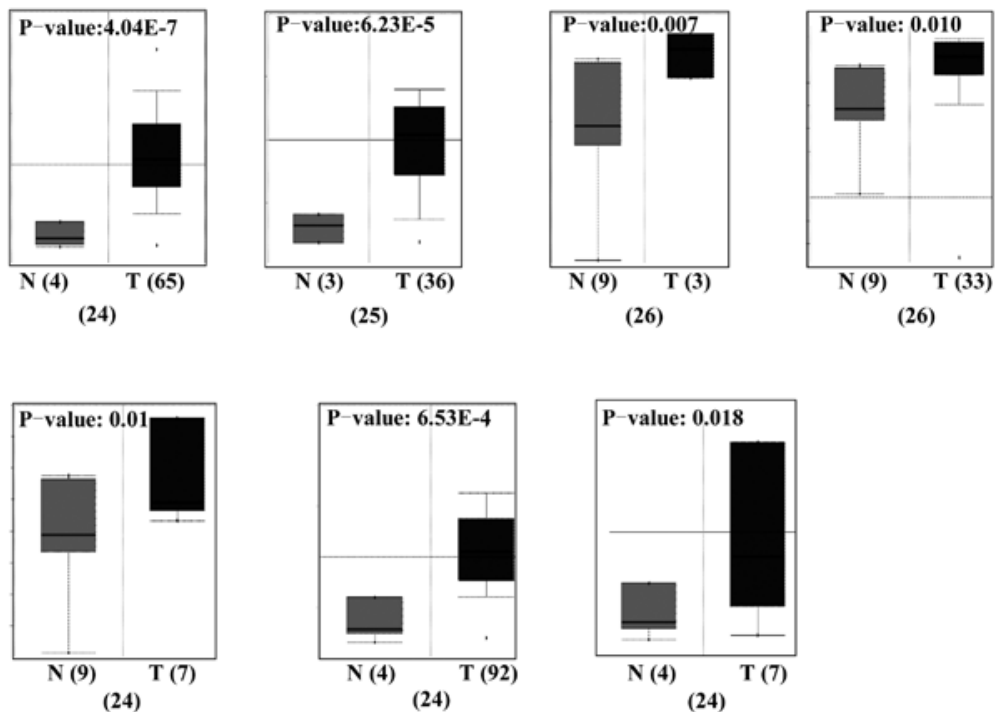

B

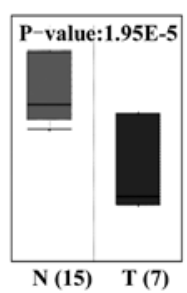

(27)

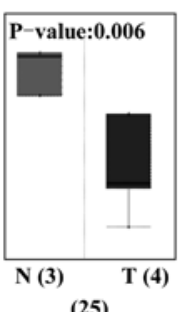

(25)

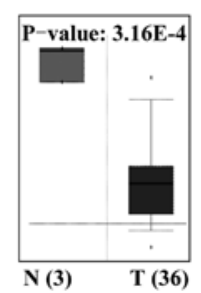

(25)

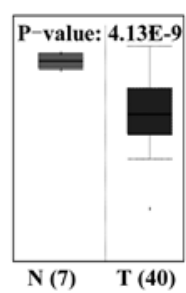

(28)

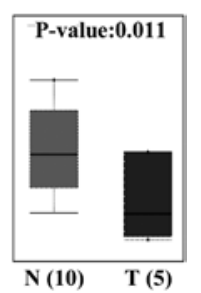

(29)

Figure 4. Expression of HAX1 and hSav1 in breast cancer. The Oncomine database was used to analyze previously published microarray data (24-29). (A) Levels of HAX1 mRNA are increased in human breast cancers when compared to normal breast tissue. (B) hSavl mRNA levels are decreased in breast cancers when compared to normal breast tissue. All data, including P-values, were calculated from the Oncomine database. 
function of HAX1 after knockdown of endogenous hSav1 in MCF-7 cells. Significantly, knockdown the endogenous hSav1 enhanced the anti-apoptotic function of HAX1, either in the Sub-G1 assay (Fig. 3D) ( 21 vs. $\sim 10 \% ; n=3, P<0.05)$ or in the Annexin V/PI assay (Fig. 3E) $(\sim 29$ vs. $\sim 16 \%$; $n=3, \mathrm{P}<0.05)$. These findings were further confirmed by the subsequent protein changes shown in Fig. 3C and F.

HAXl and hSavl expression in breast cancer. In the present study, we queried the Oncomine database to systematically assess relative gene expression levels of HAX1 and hSav1 in breast cancer. We found that HAX1 expression was significantly higher in breast cancer compared with normal breast tissue in seven microarray expression studies (Fig. 4A). Similar analysis of hSav1 showed that it was also significantly down-regulated in five microarray expression studies (Fig. 4B).

\section{Discussion}

In the present study, we identified HAX1 as a novel binding partner for hSav1. We also showed that HAX1 is critical for protecting against apoptosis and that hSav1 attenuated the anti-apoptotic effects of $\mathrm{HAX} 1$ in $\mathrm{H}_{2} \mathrm{O}_{2}$-induced apoptosis in MCF-7 cells. Finally, we used the Oncomine database to show that HAX1 expression was up-regulated while hSav1 was down-regulated in breast cancer samples.

Studies in Drosophila have uncovered a growth regulatory pathway mediated by HPO, the Drosophila ortholog for the MST (mammalian sterile 20-like kinase) family, which promotes apoptosis and restricts cell proliferation in conjunction with the scaffold protein SAV $(6,7,12)$ as part of the SWH pathway. It was previously shown that SAV regulates apoptosis by virtue of its ability to modulate the levels of DIAP1; loss of the gene $S a v$ increased DIAP1 levels; the human ortholog of Drosophila Sav, hSavl, codes for an ubiquitously expressed protein consisting of two WW domains and a coiled-coil region with a molecular mass of $\sim 45 \mathrm{kDa}$ (30).

We showed that HAX1, a 35-kDa mitochondrial outer membrane protein, binds to hSav1. HAX1 is known to participate in both death receptor and mitochondria-mediated apoptosis pathways (16) and functions as an endogenous anti-apoptotic molecule. We found that the C-terminal 80 -amino acid sequence (208-279) of HAX1, consisting of a transmembrane domain, is required for binding to hSav1.

Other studies have suggested that HAX1 is involved in both stabilization of the mitochondrial membrane potential and in protection against apoptosis $(31,32)$. Permeabilization of mitochondrial membranes is often a rate-limiting process in apoptotic cell death $(33,34)$. It has been reported that HAX1 regulates carcinoma cell migration and invasion via clathrinmediated endocytosis of integrin $\alpha v \beta 6$ and progression of oral cancer has been associated with enhanced expression of $\alpha v \beta 6$ and HAX1 proteins in patient tissue (35). In the present study, the HAX1 protected against cell death in MCF-7 breast cancer cells. We also found that HAX1 is up-regulated in breast cancer samples compared to normal breast tissues by searching the Oncomine database, and therefore it may also play an important role in breast cancer progression. Recently the Hippo signaling pathway was reported to be a critical regulator of mammalian liver growth and a potent suppressor of liver tumor formation (36). The mammalian Hippo-Salvador pathway restricts the proliferation of hepatic oval cells and thereby controls liver size and prevents the development of oval cell-derived tumors (37). Both of these studies suggest that hSav1 may be a potent tumor repressor of liver tumor formation. We also showed that hSav1 expression was down-regulated in breast cancer and it attenuated the anti-apoptotic function of HAX1 in MCF-7 cells. Taken together, the deregulation of both hSav1 and HAX1 may play a role during breast tumorigenesis.

\section{Acknowledgements}

We sincerely thank Dhan V. Kalvakolanu for editing this manuscript. This study was supported by funds from the Program for New Century Excellent Talents in University (NCET) (no. NCET-04-0699), the National Natural Science Foundation of China (NSFC) (nos. 30872472, 30800569 and 30973496), the Natural Science Foundation of Hubei Province (2008CDB174 and 2009CDB239) and the National Basic Research Program of China (973: no. 2009CB521802).

\section{References}

1. Bennett $\mathrm{F}$ and Harvey K: Fat cadherin modulates organ size in Drosophila via the Salvador/Warts/Hippo signaling pathway. Curr Biol 16: 2101-2110, 2006.

2. Silva E, Tsatskis Y, Gardano L, Tapon $\mathrm{N}$ and McNeill H: The tumor-suppressor gene fat controls tissue growth upstream of expanded in the hippo signaling pathway. Curr Biol 16: 2081-2089, 2006.

3. Hamaratoglu F, Willecke M, Kango-Singh M, et al: The tumoursuppressor genes NF2/Merlin and Expanded act through Hippo signalling to regulate cell proliferation and apoptosis. Nat Cell Biol 8: 27-36, 2006.

4. Tapon N, Harvey K, Bell D, et al: Salvador promotes both cell cycle exit and apoptosis in Drosophila and is mutated in human cancer cell lines. Cell 110: 467-478, 2002.

5. Guo C, Tommasi S, Liu L, Yee J, Dammann R and Pfeifer G: RASSF1A is part of a complex similar to the Drosophila Hippo/ Salvador/Lats tumor-suppressor network. Curr Biol 17: 700-705, 2007.

6. Udan R, Kango-Singh M, Nolo R, Tao C and Halder G: Hippo promotes proliferation arrest and apoptosis in the Salvador/Warts pathway. Nat Cell Biol 5: 914-920, 2003.

7. Harvey K, Pfleger C and Hariharan I: The Drosophila Mst ortholog, hippo, restricts growth and cell proliferation and promotes apoptosis. Cell 114: 457-467, 2003.

8. Huang J, Wu S, Barrera J, Matthews K and Pan D: The Hippo signaling pathway coordinately regulates cell proliferation and apoptosis by inactivating Yorkie, the Drosophila homolog of YAP. Cell 122: 421-434, 2005.

9. Oh $\mathrm{H}$ and Irvine K: In vivo regulation of Yorkie phosphorylation and localization. Development 135: 1081-1088, 2008.

10. Harvey K and Tapon N: The Salvador-Warts-Hippo pathway - an emerging tumour-suppressor network. Nat Rev Cancer 7: 182-191, 2007.

11. Kango-Singh M, Nolo R, Tao C, et al: Shar-pei mediates cell proliferation arrest during imaginal disc growth in Drosophila. Development 129: 5719-5730, 2002.

12. Pantalacci S, Tapon N and Léopold P: The Salvador partner Hippo promotes apoptosis and cell-cycle exit in Drosophila. Nat Cell Biol 5: 921-927, 2003.

13. Wu S, Huang J, Dong J and Pan D: Hippo encodes a Ste-20 family protein kinase that restricts cell proliferation and promotes apoptosis in conjunction with salvador and warts. Cell 114: 445-456, 2003.

14. Dong J, Feldmann G, Huang J, et al: Elucidation of a universal size-control mechanism in Drosophila and mammals. Cell 130: $1120-1133,2007$ 
15. Suzuki Y, Demoliere C, Kitamura D, Takeshita H, Deuschle U and Watanabe T: HAX1, a novel intracellular protein, localized on mitochondria, directly associates with HS1, a substrate of Src family tyrosine kinases. J Immunol 158: 2736-2744, 1997.

16. Sharp T, Wang H, Koumi A, et al: K15 protein of Kaposi's sarcoma-associated herpesvirus is latently expressed and binds to $\mathrm{HAX} 1$, a protein with antiapoptotic function. J Virol 76 802-816, 2002

17. Cilenti L, Soundarapandian M, Kyriazis G, et al: Regulation of HAX1 anti-apoptotic protein by $\mathrm{Omi} / \mathrm{HtrA} 2$ protease during cell death. J Biol Chem 279: 50295-50301, 2004.

18. Mirmohammadsadegh A, Tartler U, Michel G, et al: HAX1, identified by differential display reverse transcription polymerase chain reaction, is overexpressed in lesional psoriasis. $\mathrm{J}$ Invest Dermatol 120: 1045-1051, 2003.

19. Shaw J and Kirshenbaum L: HAX1 represses postmitochondrial caspase-9 activation and cell death during hypoxia-reoxygenation. Circ Res 99: 336-338, 2006.

20. Han Y, Chen Y, Liu Z, et al: Overexpression of HAX1 protects cardiac myocytes from apoptosis through caspase-9 inhibition. Circ Res 99: 415-423, 2006.

21. Luo X, Li Z, Yan Q, et al: The human WW45 protein enhances MST1-mediated apoptosis in vivo. Int J Mol Med 23: 357-362, 2009.

22. Luo X, Zhou Y, Tao D, et al: Usefulness of oral mucosal epithelial cell apoptosis rate in nutritional assessment. Nutrition 22 : 1032-1038, 2006

23. Rhodes DR, Kalyana-Sundaram S, Mahavisno V, et al: Oncomine 3.0: genes, pathways, and networks in a collection of 18,000 cancer gene expression profiles. Neoplasia 9: 166-180, 2007.

24. Sorlie T, Tibshirani R, Parker J, et al: Repeated observation of breast tumor subtypes in independent gene expression data sets. Proc Natl Acad Sci USA 100: 8418-8423, 2003.

25. Perou CM, Sørlie T, Eisen MB, et al: Molecular portraits of human breast tumours. Nature 406: 747-752, 2000.

26. Radvanyi L, Singh-Sandhu D, Gallichan S, et al: The gene associated with trichorhinophalangeal syndrome in humans is overexpressed in breast cancer. Proc Natl Acad Sci USA 102: 11005-11010, 2005.
27. Karnoub AE, Dash AB, Vo AP, et al: Mesenchymal stem cells within tumour stroma promote breast cancer metastasis. Nature 449: 557-563, 2007.

28. Richardson AL, Wang ZC, De Nicolo A, et al: X chromosomal abnormalities in basal-like human breast cancer. Cancer Cell 9: 121-132, 2006

29. Turashvili G, Bouchal J, Baumforth K, et al: Novel markers for differentiation of lobular and ductal invasive breast carcinomas by laser microdissection and microarray analysis. BMC Cancer 7: 55,2007

30. Valverde P: Cloning, expression, and mapping of hWW45, a novel human WW domain-containing gene. Biochem Biophys Res Commun 276: 990-998, 2000.

31. Klein C, Grudzien M, Appaswamy G, et al: HAX1 deficiency causes autosomal recessive severe congenital neutropenia (Kostmann disease). Nat Genet 39: 86-92, 2007.

32. Chao J, Parganas E, Boyd K, Hong C, Opferman J and Ihle J: Hax1-mediated processing of HtrA2 by Parl allows survival of lymphocytes and neurons. Nature 452: 98-102, 2008.

33. Green D and Kroemer G: The pathophysiology of mitochondrial cell death. Science 305: 626-629, 2004.

34. Newmeyer D and Ferguson-Miller S: Mitochondria: releasing power for life and unleashing the machineries of death. Cell 112: 481-490, 2003

35. Ramsay A, Keppler M, Jazayeri M, et al: HS1-associated protein $\mathrm{X}-1$ regulates carcinoma cell migration and invasion via clathrin-mediated endocytosis of integrin alphavbeta6. Cancer Res 67: 5275-5284, 2007.

36. Lu L, Li Y, Kim SM, et al: Hippo signaling is a potent in vivo growth and tumor suppressor pathway in the mammalian liver. Proc Natl Acad Sci USA 107: 1437-1442, 2010.

37. Lee KP, Lee JH, Kim TS, et al: The Hippo-Salvador pathway restrains hepatic oval cell proliferation, liver size, and liver tumorigenesis. Proc Natl Acad Sci USA 107: 8248-8253, 2010. 